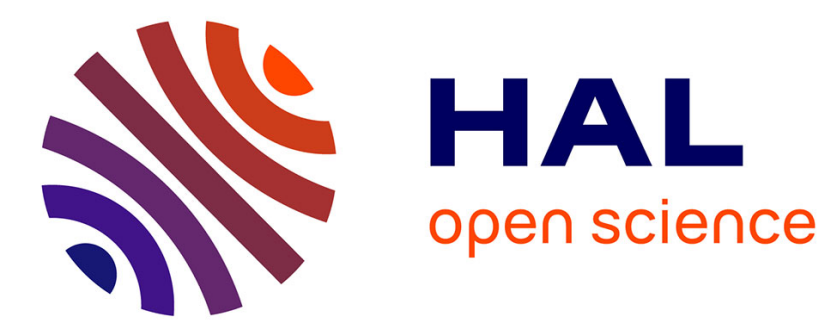

\title{
Structure cristalline de la schmitterite synthétique UTeO5
}

\author{
Georges Meunier, Jean Galy
}

\section{To cite this version:}

Georges Meunier, Jean Galy. Structure cristalline de la schmitterite synthétique UTeO5. Acta crystallographica Section B: Structural crystallography and crystal chemistry, 1973, 29 (6), pp.1251-1255. 10.1107/S0567740873004334 . hal-00151791

\section{HAL Id: hal-00151791 \\ https://hal.science/hal-00151791}

Submitted on 5 Jun 2007

HAL is a multi-disciplinary open access archive for the deposit and dissemination of scientific research documents, whether they are published or not. The documents may come from teaching and research institutions in France or abroad, or from public or private research centers.
L'archive ouverte pluridisciplinaire HAL, est destinée au dépôt et à la diffusion de documents scientifiques de niveau recherche, publiés ou non, émanant des établissements d'enseignement et de recherche français ou étrangers, des laboratoires publics ou privés. 
Acta Cryst. (1973). B29, 1251

\title{
Structure Cristalline de la Schmitterite Synthétique $\mathrm{UTeO}_{5}$
}

\author{
Par Georges Meunier et Jean Galy \\ Service de Chimie Minérale Structurale de l'Université de Bordeaux I, assucié au CNRS, \\ 351 cours de la Libération, 33 405-Talence, France
}

(Reçu le 15 janvier 1973, accepté le 16 février 1973)

\begin{abstract}
Synthetic schmitterite $\mathrm{UTeO}_{5}$ crystallizes in the orthorhombic system. The parameters are $a=10 \cdot 161$, $b=5.363$ and $c=7.862 \AA$; the space group is $P c a 2_{1}$. The structure is built up of infinite chains $\left[\mathrm{UO}_{5}\right]_{n}^{4 n-}$ of pentagonal bipyramids sharing edges and running parallel to the $c$ axis. The tellurium (+IV) atoms, coordinated on one side to four oxygen atoms, maintain the rigidity of the network. Structural relations with $\alpha-\mathrm{U}_{3} \mathrm{O}_{8}$ and $\mathrm{UVO}_{5}$ are discussed. A hypothetical structure with a $\mathrm{U}_{2} \mathrm{M}^{2+} \mathrm{O}_{7}$ formula is proposed.
\end{abstract}

C'est dans le Sonora, état du nord-ouest du Mexique, dans les mines de Moctezuma que Gaines (1969) découvrait un minerai inédit d'uranium et de tellure. Il baptisait ce minerai la cliffordite et lui attribuait la formule $\mathrm{UTe}_{3} \mathrm{O}_{8}$.

$\mathrm{Au}$ cours d'une étude chimique et radiocristallographique des systèmes $\mathrm{UO}_{2}-\mathrm{TeO}_{2}$ et $\mathrm{UO}_{3}-\mathrm{TeO}_{3}$, Galy \& Meunier (1971) ont montré que la cliffordite avait pour formule $\mathrm{UTe}_{3} \mathrm{O}_{9}$ au lieu de $\mathrm{UTe}_{3} \mathrm{O}_{8}$. Ces auteurs précisaient, dans ce même travail, les conditions de synthèse et les constantes cristallographiques d'une autre phase de formule $\mathrm{UTeO}_{5}$.

$\mathrm{UTeO}_{5}$ avait été préparé pour la première fois par Khodadad (1962).

Plus récemment, en 1971, Gaines découvrait, dans les mines de Moctezuma, une nouvelle espèce naturelle, la schmitterite. La formule chimique annoncée était $\mathrm{UTeO}_{5}$. Les paramètres de la maille et le groupe spatial concordaient avec ceux antérieurement publiés pour $\mathrm{UTeO}_{5}$ de synthèse.

Ce mémoire décrit la détermination structurale de la phase $\mathrm{UTeO}_{5}$ préparée par synthèse.

\section{Constantes cristallographiques de $\mathrm{UTeO}_{5}$}

Nous rappelons brièvement, au Tableau 1, les constantes cristallographiques de $\mathrm{UTeO}_{5}$; elles sont comparées aux valeurs annoncées par Gaines (1971) pour l'espèce naturelle.

Tableau 1. Constantes cristallographiques de $\mathrm{UTeO}_{5}$ et de la schmitterite

$\begin{array}{llc} & \mathrm{UTeO}_{5}, \text { Galy \& } & \text { Schmitterite, } \\ & \text { Meunier (1971) } & \text { Gaines (1971) } \\ a & 10,161 \pm 0,004 \AA & 7,860 \pm 0.004 \AA \\ b & 5,363 \pm 0,003 & 10,089 \pm 0,002 \\ c & 7,862 \pm 0,003 & 5,363 \pm 0,002 \\ \text { Groupes } & P c a 2_{1} \text { ou } & \\ \text { spatiaux } & P c a m & \text { Pmab } \\ d_{\text {exp }} & 6,91 \pm 0,04 \mathrm{~g} \mathrm{~cm}^{-3} & 6,878 \mathrm{~g} \mathrm{~cm}^{-3} \\ d_{x} & 6,91 & 6,91 \\ Z & 4 & 4\end{array}$

\section{Détermination de la structure cristalline}

De nombreux monocristaux de couleur jaune présentant l'aspect de prismes réguliers ont été obtenus par transport chimique à $600^{\circ} \mathrm{C}$ en tube de Vycor scellé sous vide. L'agent de transport était le tétrachlorure de tellure $\mathrm{TeCl}_{4}$, utilisé à raison de $10 \mathrm{mg}$ pour un volume de $1 \mathrm{~cm}^{3}$ du tube réactionnel.

Le monocristal choisi pour l'étude structurale avait la forme d'un parallélépipède régulier de dimensions $0,06 \times 0,04 \times 0,10 \mathrm{~mm}$; il était orienté de manière rigoureuse suivant l'axe $O z$.

Les intensités des réflexions $h k l$ ont été mesurées à l'aide d'un diffractomètre automatique Enraf Nonius CAD 3 (radiation Mo $K \alpha$, monochromateur à lame de graphite).

L'angle maximum de mesure était $\theta_{\max }=35^{\circ}$; le balayage en $\omega$ était effectué. Seules ont été retenues les réflexions $h k l$ dont l'intensité satisfaisait au test $I_{h k l}>$ $1,5 \sigma\left(I_{h k l}\right) .641$ réflexions indépendantes ont ainsi été sélectionnées. Ces intensités ont été corrigées tout d'abord du facteur de Lorentz-polarisation.

Mais étant donné la valeur importante du coefficient d'absorption linéaire $\mu(M o K \alpha)=640 \mathrm{~cm}^{-1}$, il s'avérait nécessaire de procéder à une seconde correction. Après indexation des faces du monocristal, la correction d'absorption a été effectuée à l'aide du programme $D A T A P$ 1 (Coppens, Leiserowitz \& Rabinovich, 1965). Les coefficients d'absorption massique des éléments ont été tirés de l'ouvrage de Bragg (1966).

Les facteurs de diffusion des atomes d'uranium, de tellure et d'oxygène ont été relevés dans les tables publiées par Cromer \& Waber (1965). Les deux premiers facteurs ont été corrigés de la dispersion anomale $\left[\Delta f^{\prime}=9,19, \Delta f^{\prime \prime}=9,09\right.$ pour l'uranium; $\Delta f^{\prime}=-0,69$, $\Delta f^{\prime \prime}=2,00$ pour le tellure; Cromer (1965)].

Les calculs ont été effectués sur I.B.M. 360-44 à l'aide de programmes mis au point au laboratoire par M. Saux.

Les positions atomiques de l'uranium et du tellure ont été obtenues à partir de la fonction de Patterson tridimensionnelle $P(u, v, w)$ : 
$\mathrm{U}$ en $4(a): 0,0,0$

Te en $4(d): x, y, \frac{1}{4}$ avec $x=0,25$ et $y=0,37$.

Un premier calcul d'affinement utilisant les blocs diagonaux, dans le cas du groupe spatial Pcam, n'a pas permis d'abaisser l'indice $R$ en-dessous de 0,45 . Nous avons donc abandonné le groupe centrosymétrique Pcam pour $P c a 2_{1}$. Les tests statistiques de Wilson (1949), de Howells, Phillips \& Rogers (1950) et de Srinivasan (1960) permettent d'ailleurs de prévoir une structure non centrée.

Les calculs ont donc été repris en mettant les atomes lourds dans la position générale $4(a): x, y, z$. La coordonnée $z$ de l'uranium était fixée à la valeur $z=0$ afin de donner une origine à la structure suivant la direction de l'axe $O z$. Après quelques cycles d'affinement, l'indice $R$ est abaissé jusqu'à 0,10 .
Une synthèse de Fourier $\varrho(x, y, z)$ a permis de déduire des valeurs obtenues les coordonnées atomiques des cinq atomes d'oxygène.

Un affinement de la structure a alors été entrepris faisant intervenir la matrice complète (Busing, Martin \& Levy, 1962). L'indice $R$ est alors de 0,058 .

Les positions atomiques et les facteurs d'agitation thermique de l'uranium, du tellure et des oxygènes sont rassemblés au Tableau 2. Les distances interatomiques et les principaux angles de liaison figurent au Tableau 3.*

* La liste des facteurs de structure observés et calculés a été déposée au dépôt d'archives de la National Lending Library, Angleterre (Supplementary Publication No. SUP 30076). Des copies peuvent être obtenues en s'adressant à: The Executive Secretary, International Union of Crystallography, 13 White Friars, Chester CH 11 NZ, Angleterre.

Tableau 2. Coordonnées atomiques et facteurs d'agitation thermique

\begin{tabular}{|c|c|c|c|c|c|c|c|}
\hline & $\mathrm{U}$ & $\mathrm{Te}$ & $\mathrm{O}(1)$ & $\mathrm{O}(2)$ & $\mathrm{O}(3)$ & $\mathrm{O}(4)$ & $\mathrm{O}(5)$ \\
\hline$x$ & $0,0148(1)$ & $0,250(2)$ & $0,214(3)$ & $0,103(4)$ & $0,392(5)$ & 0,615 (3) & $0,590(3)$ \\
\hline$y$ & $0,0302(2)$ & 0,3662 (4) & 0,270 (5) & $0,161(6)$ & $0,120(8)$ & $0,241(6)$ & $0,306(6)$ \\
\hline & 0,0000 & $0,247(2)$ & $-0,026(4)$ & $0,284(6)$ & 0,233 (7) & 0,027 (4) & 0,531 (4) \\
\hline$B(\AA)^{2}$ & & & $0,82(53)$ & $0,30(62)$ & $0,84(80)$ & 0,75 (55) & $1,00(60)$ \\
\hline $\begin{array}{l}\beta_{11} \\
\beta_{22}\end{array}$ & $\begin{array}{l}0,0020(1) \\
0,0080(3)\end{array}$ & $\begin{array}{l}0,0008(1) \\
0,0029(5)\end{array}$ & & & & & \\
\hline$\beta_{33}$ & $0,0044(2)$ & $0,0028(4)$ & & & & & \\
\hline$\beta_{12}$ & $-0,0004(2)$ & $-0,0009$ (17) & & & & & \\
\hline$\beta_{23}$ & $-0,0001(10)$ & $0,0001(2)$ & & & & & \\
\hline$\beta_{13}$ & $-0,0021(10)$ & $-0,0029(14)$ & & & & & \\
\hline
\end{tabular}

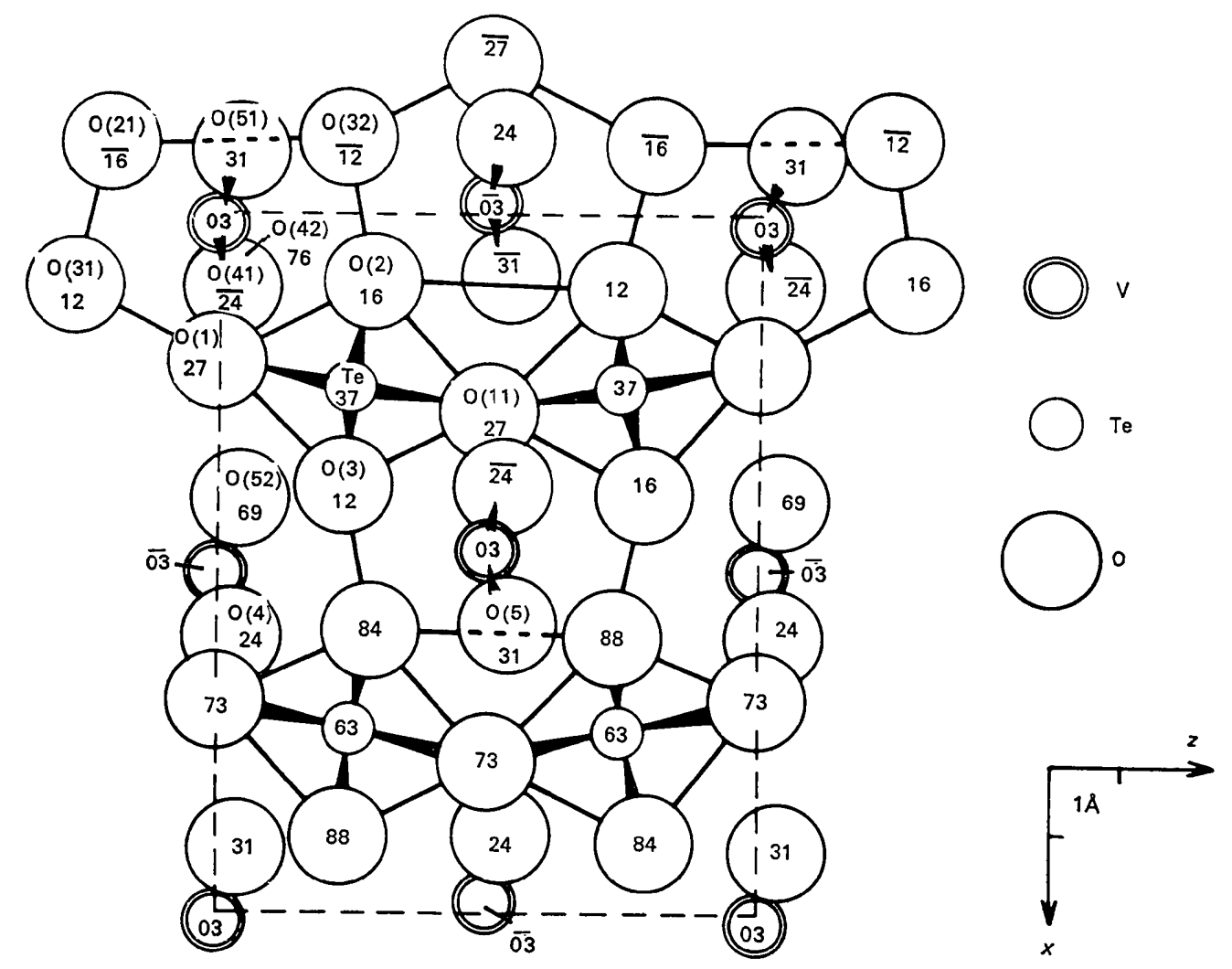

Fig. 1. Projection de la structure sur le plan (010). 


\section{Description de la structure et discussion}

La projection de la structure sur le plan (010) est donnée à la Fig. 1. Les cotes $y$ sont indiquées à l'intérieur des cercles figurant les atomes $(100 y)$.

L'atome d'uranium est lié à 7 atomes d'oxygène; cette coordinence 7 est du type bipyramidal à base pentagonale (Fig. 2). Cinq atomes d'oxygène, à une distance moyenne $\mathrm{U}-\mathrm{O}=2,39 \AA$, forment un pentagone plan légèrement distordu. De part et d'autre de ce plan, deux oxygènes à des distances beaucoup plus courtes $\mathrm{U}-\mathrm{O}(41)=1,79 \AA$ et $\mathrm{U}-\mathrm{O}(51)=1,84 \AA$ forment avec l'uranium un groupement uranyle $\mathrm{UO}_{2}^{2+}$.

Cet environnement est en tout point comparable avec celui rencontré dans $\mathrm{K}_{3} \mathrm{UO}_{2} \mathrm{~F}_{5}$ (Zachariasen, 1954), dans $\mathrm{H}_{2} \mathrm{U}_{3} \mathrm{O}_{10}$ (Siegel, Viste, Hoekstra \& Tani, 1972) et dans $\mathrm{UVO}_{5}$ (Chevalier \& Gasperin, 1970).

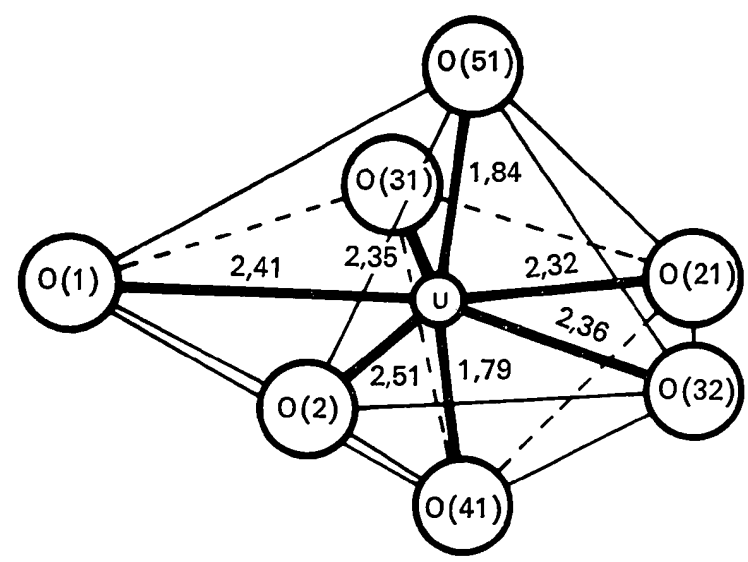

Fig. 2. Environnement oxygéné de l'uranium.

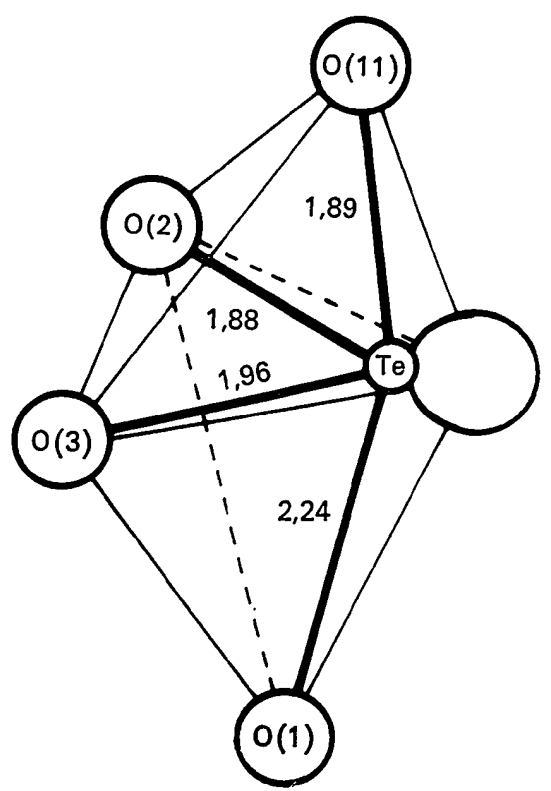

Fig. 3. Environnement oxygéné du tellure.
Tableau 3. Distances interatomiques (erreur maximum $\pm 0,03 \AA)$ et angles de liaison principaux $\mathrm{O}-\mathrm{Te}-\mathrm{O}$

$\begin{array}{llll}\text { U-O(41) } & 1,79 \AA & \text { Te-O(2) } & 1,88 \AA \\ \text { U-O(51) } & 1,84 & \text { Te-O(11) } & 1,89 \\ \text { U-O(21) } & 2,32 & \text { Te-O(3) } & 1,96 \\ \text { U-O(31) } & 2,35 & \text { Te-O(1) } & 2,24 \\ \text { U-O(32) } & 2,36 & \text { Te-O(52) } & 2,95 \\ \text { U-O(1) } & 2,41 & \text { Te-O(42) } & 3,05 \\ \text { U-O(2) } & 2,51 & & \\ \text { O(1)-O(2) } & 2,75 & \text { O(2)-O(3) } & 2,97 \\ \text { O(1)-O(31) } & 2,32 & \text { O(1)-O(11) } & 4,00 \\ \text { O(21)-O(31) } & 3,54 & \text { O(1)-O(2) } & 2,75 \\ \text { O(2)-O(32) } & 2,65 & \text { O(1)-O(3) } & 2,84 \\ \text { O(21)-O(31) } & 2,65 & \text { O(2)-O(11) } & 2,46 \\ \text { O(32)-O(41) } & 2,86 & \text { O(3)-O(11) } & 2,32 \\ \text { O(21)-O(41) } & 2,96 & & \\ \text { O(1)-O(41) } & 2,95 & & \\ \text { O(2)-O(41) } & 2,96 & & \\ \text { O(31)-O(41) } & 3,02 & & \\ \text { O(32)-O(51) } & 2,79 & & \\ \text { O(21)-O(51) } & 3,17 & & \\ \text { O(1)-O(51) } & 3,13 & & \\ \text { O(2)-O(51) } & 2,90 & & \\ \text { O(31)-O(51) } & 3,25 & & \end{array}$

Angles principaux $\mathrm{O}-\mathrm{Te}-\mathrm{O}$ (erreur maximum $\pm 1^{\circ}$ ) $\mathrm{O}(2)-\mathrm{Te}-\mathrm{O}(3) \quad 101,5^{\circ} \quad \mathrm{O}(1)-\mathrm{Te}-\mathrm{O}(11) \quad 150,8^{\circ}$

L'atome de tellure comporte la coordinence 4 (Fig. 3). Quatre oxygènes sont situés tous d'un même côté du tellure, à des distances $\mathrm{Te}-\mathrm{O}$ comprises entre 1,88 et $2,24 \AA$. Ce polyèdre $\left[\mathrm{TeO}_{4}\right]$ est à rapprocher de celui mis en évidence par Leciejewicz (1961) et Lindqvist (1968) dans la paratellurite $\mathrm{TeO}_{2}$, par Meunier \& Galy (1971) dans les phases $\mathrm{MTe}_{3} \mathrm{O}_{8}$ où $\mathrm{M}$ était le titane, le zirconium, le hafnium et l'étain, et également dans la cliffordite $\mathrm{UTe}_{3} \mathrm{O}_{9}$ (Galy \& Meunier, 1971).

L'environnement du tellure peut être décrit comme une bipyramide à base triangulaire, l'un des sommets du triangle équatorial étant constitué par le doublet non engagé du tellure. Il faut remarquer que trois distances sont inférieures à $2 \AA$ et que la quatrième est égale à $2,24 \AA$. Le tellure marque donc ici une certaine tendance à la coordinence $3+1$, tendance que l'on retrouve dans la mackayite $\mathrm{Fe}(\mathrm{OH})\left[\mathrm{Te}_{2} \mathrm{O}_{5}\right]$ étudiée par Pertlik (1969) et la poughite $\mathrm{Fe}_{2}\left(\mathrm{TeO}_{3}\right)_{2} \mathrm{SO}_{4}$. $3 \mathrm{H}_{2} \mathrm{O}$ (Pertlik, 1971).

L'agencement de la structure se réalise tout d'abord au niveau des bipyramides pentagonales $\left(\mathrm{UO}_{7}\right)$; cellesci, accolées les unes aux autres par l'intermédiaire d'arêtes communes, forment des chaînes de formule $\left(\mathrm{UO}_{5}\right)_{n}^{4 n-}$ parallèles à la direction de l'axe $\mathrm{Oz}$. Ces chaînes parallèles se répètent dans la direction $O y$ avec une période $\mathbf{b}$ et dans la direction $O x$ avec une période a/2. Le tellure assure la cohésion du réseau en pontant les chaînes entre elles.

\section{Relations structurales}

A la Fig. 4(a) est représentée la structure idéalisée de $\mathrm{U}_{3} \mathrm{O}_{8} \alpha$ d'après Andresen (1958). Cette structure consiste en feuillets plissés formés de bipyramides penta- 
gonales $\left(\mathrm{UO}_{7}\right)$ mettant en commun des arêtes dans la direction $O x$ et des sommets dans la direction $O y$. Ces feuillets parallèles au plan $x O z$ sont liés les uns aux autres par mise en commun du cinquième sommet de la base pentagonale. L'arête opposée à ce sommet est commune à un octaèdre $\mathrm{UO}_{6}$ inséré entre les feuillets. Les octaèdres, comme d'ailleurs les bipyramides pentagonales, forment des files infinies dans la direction $O y$.

Les feuillets plissés sont schématisés en projection à la Fig. 4(b). La période de répétition suivant la direction $O x$ est u. Comme l'a développé récemment Jahnberg (1971), on peut faire subir à deux feuillets consécutifs un glissement $\mathbf{a} / 2$; le motif obtenu est alors représenté à la Fig. 4(c). Les nouveaux feuillets peuvent alors être connectés dans la direction $O z$ par des files infinies d'octaèdres $\mathrm{UO}_{6}$ selon la disposition indiquée à la Fig. $4(d)$, qui est celle du réseau de $\mathrm{UVO}_{5}$. Les similitudes entre les structures $\mathrm{UVO}_{5}$ et $\mathrm{UTeO}_{5}$ [figure idéalisée en $4(e)$ ] apparaissent immédiatement. Les positions des atomes d'uranium sont comparables (même environnement également) et le tellure se place sensiblement dans la même position que le vanadium. Cependant, la présence du doublet non engagé du tellure ne permet pas la présence, à ce niveau, d'un atome d'oxygène comme dans $\mathrm{UVO}_{5}$. La formule devrait donc être en fait $\mathrm{UTeO}_{4}$. Celle de la phase étudiée étant en réalité $\mathrm{UTeO}_{5}$, un changement doit nécessairement se produire dans les feuillets de bipyramides pentagonales. Il résultera de la rupture de la liaison entre bipyramides pentagonales suivant l'axe $O y$; il est illustré à la Fig. 5. A la Fig. 5(a) sont représentées les chaînes infinies $\left(\mathrm{UO}_{6}\right)$ de $\mathrm{UVO}_{5}$. Leur projection apparaît à la Fig. $5(b)$. La Fig. 5(c) montre que dans $\mathrm{UTeO}_{5}$ il n'y a plus selon l'axe des chaînes de sommets communs; ce simple fait explique que l'on conserve la formule $\mathrm{ABO}_{5}$ malgré le remplacement du vanadium par le tellure. La projection idéalisée selon l'axe $O y$ des nouvelles bipyramides pentagonales est donnée à la Fig. $5(d)$. La structure idéalisée de $\mathrm{UTeO}_{5}$ apparaît à la Fig. 4(e).

Ces remarques résultant de la comparaison entre les structures de $\mathrm{UTeO}_{5}$ et $\mathrm{UVO}_{5}$ nous ont amené à proposer une nouvelle structure [Fig. $4(f)$ ] en partant d'une disposition de chaînes $\left(\mathrm{UO}_{5}\right)_{n}^{4 n-}$ identique à celle de $\mathrm{U}_{3} \mathrm{O}_{8} \alpha$. L'uranium placé en site octaédrique et l'oxygène en position axiale sont remplacés, comme dans $\mathrm{UTeO}_{5}$, par un élément comportant comme le tellure un doublet non engagé. La formule cristallographique obtenue est $\mathrm{U}_{2} \mathrm{MO}_{7}$; tenant compte du degré d'oxydation + VI de l'uranium, l'élément $M$ est donc au degré d'oxydation + II. La structure hypothétique proposée à la Fig. $4(f)$ pourrait donc correspondre à une phase ' $\mathrm{U}_{2} \mathrm{Sn}^{2+} \mathrm{O}_{7}$ ' ou ' $\mathrm{U}_{2} \mathrm{~Pb}^{2+} \mathrm{O}_{7}$ '

\section{Références}

ANDResen, A. F. (1958). Acta Cryst. 11, 612-614.

BraGG, W. L. (1966). The Crystalline State. Vol. 1, p. 327. London: Bell.

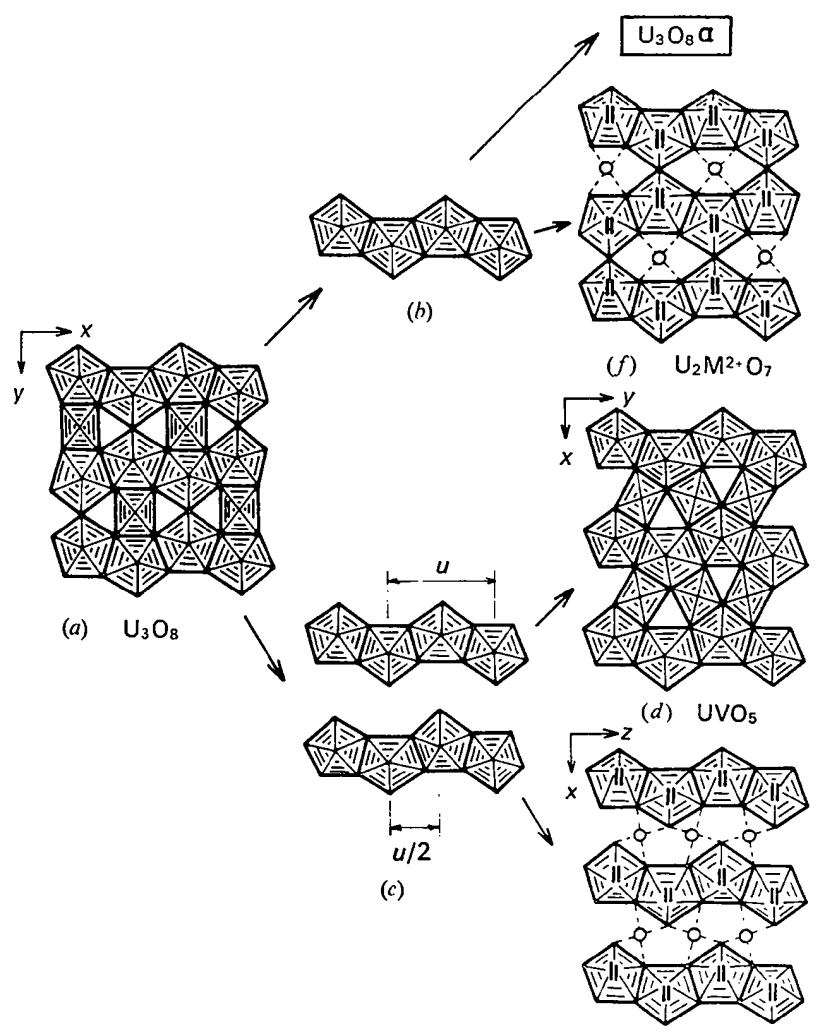

(e) $\mathrm{UTeO}_{5}$

Fig. 4. Relations structurales entre $\mathrm{U}_{3} \mathrm{O}_{8} \alpha, \mathrm{UVO}_{5}, \mathrm{UTeO}_{5}$ et $\mathrm{U}_{2} \mathrm{M}^{2+} \mathrm{O}_{\text {, }}$.

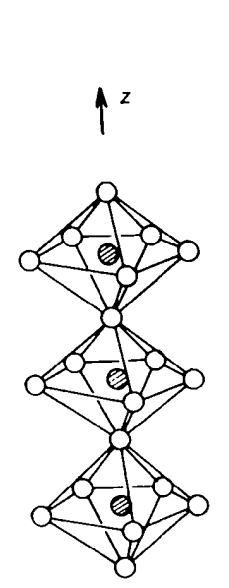

(a) $\mathrm{UVO}_{5}$

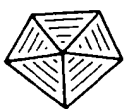

(b)

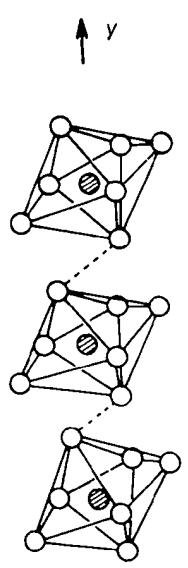

(c) $\mathrm{UTeO}_{5}$

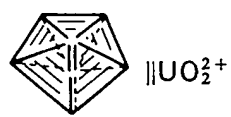

(d)
Fig. 5. Chaînes d'uranium dans $\mathrm{UVO}_{5}$ et $\mathrm{UTeO}_{5}$. 
Busing, W. R., Martin, K. O. \& Levy, H. A. (1962). ORFLS. Report ORNL-TM-305, Oak Ridge National Laboratory, Oak Ridge, Tennessee.

Chevalier, R. \& Gasperin, M. (1970). Bull. Soc. Fr. Minér. Crist. 93, 18-22.

Coppens, P., Leiserowitz, L. \& Rabinovich, D. (1965). $D A T A P 1$. Institute of Chemistry, Uppsala; Oak Ridge National Laboratories, Oak Ridge, Tennessee.

Cromer, T. (1965). Acta Cryst. 18, 17-27.

Cromer, T. \& Waber, J. T. (1965). Acta Cryst. 18, 104-109.

Gaines, R. V. (1969). Amer. Min. 54, 697-701.

Gaines, R. V. (1971). Amer. Min. 56, 411-415.

Galy, J. \& Meunier, G. (1971). Acta Cryst. B27, 608-616.

Howells, E. R., Phillips, D. C. \& Rogers, D. (1950). Acta Cryst. 3, 210-214.
JaHnberg, L. (1971). Chem. Commun. p. 13.

Khodadad, P. (1962). C.R. Acad. Sci. Paris, 255, 16171618.

LECIEJEWICZ, J. (1961). Z. Kristallogr. 116, 345-353.

LindQvist, O. (1968). Acta Chem. Scand. 22, 977-982.

Meunier, G. \& Galy, J. (1971). Acta Cryst. B27, 602-608.

Pertlik, F. (1969). Tschermaks Min. Petrogr. Mitt. 13, 219-232.

Pertlik, F. (1971). Tschermaks Min. Petrogr. Mitt. 15, 279-290.

Siegel, S., Viste, A., Hoekstra, H. R. \& TANi, B. (1972) Acta Cryst. B 28, 117-121.

Srinivasan, R. (1960). Acta Cryst. 13, 388-394.

Wilson, A. J. C. (1949). Acta Cryst. 2, 318-321.

Zachariasen, W. H. (1954). Acta Cryst. 7, 783-787. 